\title{
The COVID-19 Pandemic Experience in Multiple Sclerosis: The Good, the Bad and the Neutral
}

\author{
Hannah Morris-Bankole (D) - Aileen K. Ho ic
}

Received: November 30, 2020 / Accepted: March 6, 2021 / Published online: April 15, 2021

(C) The Author(s) 2021

\begin{abstract}
Introduction: While the current COVID-19 pandemic has affected the lives of many, there is a paucity of information on the impact on people with multiple sclerosis (MS). This study sought to gain insight into the impact of the current situation on people with MS and the factors that influence this.

Methods: 324 MS patients participated in this online cross-sectional survey during the COVID-19 lockdown period. A mixed methods design was used, with quantitative information collected on MS-related factors as well as COVID-19 impact and an open-ended, qualitative response looking for reasons behind the self-reported COVID-19 impact.

Results: We found that $48 \%$ of the participants reported that COVID-19 had a neutral impact on their lives and $16 \%$ reported a positive impact. However, $36 \%$ reported a negative impact, and had greater levels of MS- and nonMS-related worries, and higher levels of bother
\end{abstract}

H. Morris-Bankole · A. K. Ho ( $\square)$

School of Psychology and Clinical Language

Sciences, University of Reading, Reading RG6 6AL, UK

e-mail: a.k.ho@reading.ac.uk related to psychological and cognitive symptoms and fatigue than the groups reporting a neutral or positive impact. Significant predictors of this adversely affected group were age, type of MS and presence of psychological symptoms. Antidepressant medication use, time since diagnosis, gender, location, living arrangements or employment status did not predict impact. Open-ended responses explaining personal COVID-19 impact indicate that coping strategies may contribute to these findings. In particular, active, problem-focused approaches were reported by the majority of people who reported a positive impact, as well as a third of those who reported a neutral impact.

Conclusion: These findings suggest that younger people, those with progressive types of MS, and those with psychological symptoms are particularly vulnerable to the negative effects of a COVID-19 pandemic induced lockdown. Coping strategies provide further insight into these findings with those reporting active problem-focused approaches seemingly faring better than those who do not state any coping strategies. These results also have implications for understanding other like neurological conditions that share many similarities with MS and how best to direct support. 


\section{PLAIN LANGUAGE SUMMARY}

People with Multiple Sclerosis (MS) are more vulnerable to psychological disorders such as anxiety. Consequences of the COVID-19 pandemic could potentially heighten these levels due to the impact on health and healthcare. An online survey was completed by 324 people with MS during the COVID-19 pandemic to identify the level of COVID-19 impact (positive, neutral or negative) and the reasons why they felt this way. Findings show that just under half of people who completed the survey reported a neutral impact, with a negative impact reported by just over a third of participants. Having a progressive diagnosis of MS, being younger and having psychological symptoms predicted a negative impact. Coping strategies also appear to play a part in the COVID-19 experience for people with MS, with active, problem-focused strategies being an approach used by nearly all who reported a positive impact and over a third of people who reported a neutral impact. These findings can be used to direct support to the most vulnerable people within this population as well as those with other neurological conditions.

Keywords: Anxiety; Concern; Coping; COVID19; Low mood; Multiple sclerosis; Pandemic; Psychological well-being

\section{Key Summary Points}

\section{Why carry out this study?}

People with MS are more vulnerable to poor psychological well-being.

There are conflicting reports about whether anxiety has increased or remained the same during the COVID-19 pandemic.

\section{What was learned from the study?}

Just under half of our sample of people with MS reported a neutral COVID-19 impact, and over a third reported a negative impact.

Some demographic and MS-related factors (younger age, progressive types of MS and presence of psychological symptoms) can predict a negative COVID-19 impact.

Both emotion-focused and problemfocused coping strategies were instrumental in those reporting a neutral or positive impact from the COVID-19 pandemic.

\section{DIGITAL FEATURES}

This article is published with digital features, including a summary slide and plain language summary, to facilitate understanding of the article. To view digital features for this article go to https://doi.org/10.6084/m9.figshare.14161361

\section{INTRODUCTION}

The COVID-19 pandemic has had at least some level of impact on everyone as restrictions have been enforced globally. Restrictions can potentially impact many aspects of life, including finances, social and family relationships, travel, access to healthcare and leisure activities, amongst others. As a result, an increased level of mental health concerns have been observed worldwide [1]. 
The prevalence of anxiety (22\%) is disproportionately higher in people with MS than the general population [2]. It may therefore be expected that heightened levels of anxiety [3] and higher levels of MS-related concerns [4] may be reported during the current COVID-19 pandemic, in particular worries that cause concern about MS and a future living with it in the context of COVID-19 being those that have the strongest impact on the current pandemic experience [5].

Other recent research, however, has arrived at alternative conclusions, that in fact levels of anxiety have not increased as a result of the current crisis and have even improved in some cases $[6,7]$. These findings are surprising given the typical psychological presentation of MS patients, as well as the potential to exacerbate such factors further due to the nature of the COVID-19 pandemic and its potential impact on MS care [8]. These studies, however, looked at groups belonging to a particular demographic according to a specific location or diagnostic category, and may suggest that other factors may account for seemingly contradictory findings.

Differences in identification of vulnerable groups determined by demographic features have yielded varying results. Older MS patients may be more vulnerable to the effects of social isolation as a result of the pandemic [5]. However, research has also shown that younger people with MS are more vulnerable to anxiety at this time $[9,10]$. Similarly, women have been reported to be more susceptible to anxiety than men at this time [8], yet opposite results have also been found in other research, suggesting men are experiencing higher levels of anxiety during the COVID-19 pandemic [5]. This provides further evidence to suggest that there are other factors that could be at play, such as coping strategies.

Historically, research has suggested that people with MS who rely on problem-focused strategies fare better in the process of adjustment compared to those who favour emotionfocused approaches [11]. Therefore, it might be anticipated that those who are coping with the COVID-19 pandemic using less-effective coping mechanisms may be more vulnerable to a negative experience and those who use problem- focused approaches may report a positive impact.

Aside from the underlying traits and mechanisms that could underpin the reasons behind a positive, neutral or negative impact, there are also objective factors that could contribute to the level of impact.

Whilst having MS itself does not necessarily increase the risk of contracting COVID-19 [12], many people with MS are prescribed diseasemodifying treatments with immunosuppressant effects that may make the user potentially more vulnerable to contracting the virus [13]. On the other hand, it is known that some people feel that the risks of remaining on immunosuppressant therapies is favourable to risking disease relapse or progression [14]. Either way, being presented with this dilemma is an additional burden experienced by MS patients at this time.

Access and quality of care may also be affected in the wake of the pandemic and local lockdown restrictions [15] which may affect symptom management and increase MS-related concerns [16]. Therefore, it might be expected that those who report higher levels of MS-related concern and symptom bother would be more likely to endorse a negative impact of COVID-19. Selfperceived levels of symptom concern may then be responsible for less-adaptive coping methods [17]. On the other hand, it is possible that the overall impact may be perceived as neutral as there may be positive impacts due to the complex interplay of personal, household and external factors within the context of the pandemic. In particular, factors such as increased social contact with close family members could facilitate a positive experience [18].

With existing literature focusing on the COVID-19 experience as a whole, none have sought to examine the differences between those who self-report different levels of impact, either negative, neutral, or even positive. There are several factors warranting further investigation that could identify who is most vulnerable to a negative experience, both in terms of demographics and MS status, as well as any selfreported reasons for COVID-19 impact that may differentiate between those who experience a negative impact when compared to those who experience a neutral or positive impact. 
Based on what is already known about the experience of MS generally and the current literature on the impact of COVID-19 on people with MS, we sought to understand the level of impact of COVID-19 experienced by people with MS and the factors that could be contributing to this that might help to explain any discrepancies in recent findings.

To build on what is currently known, we took a more direct approach by asking participants a single question about the overall impact of COVID-19 (negative, neutral or positive) to provide an assessment of the ensuing consequence on their lives. An open ended follow-up question asking participants to provide insight into the circumstances that led to the negative, neutral or positive COVID-19 impact they experienced gave participants the opportunity to spontaneously raise relevant contributing factors. This, along with regression data on demographic factors identifying those who are most vulnerable to a negative impact, could be used to identify helpful coping strategies as stated by the positive and possibly neutral groups to direct and shape support to those who are not faring so well at this time.

As these multifaceted consequences of the pandemic and lockdown impact both MS-related and non-MS-related elements of everyday life, we expected that on balance, the impact of COVID19 would be largely neutral in the majority of people with MS, with only a subset of individuals experiencing a negative impact. We also investigated whether demographic and MS-related variables in particular might predict those who report a negative impact of COVID-19.

\section{METHODS}

\section{Participants}

324 people (273 females, 51 males) with MS (68\% relapsing remitting, $8 \%$ primary progressive, $16 \%$ secondary progressive and $8 \%$ clinically isolated syndrome or unsure) aged between 20 and $75(M=47.66, \mathrm{SD}=12.19)$ responded from the UK (40\%), USA (34\%), Australia (3\%) and other countries (23\%). The age range of people with relapse-remitting MS was 20-75 ( $M=45.67$,
$\mathrm{SD}=11.82)$ and for progressive types of MS was 30-74 with a slightly higher average age $(M=55.95, \mathrm{SD}=10.36)$.

The inclusion criteria for participation were a self-reported diagnosis of MS (or responding in behalf of someone with MS; $n=4$ ) and being over the age of 18. Exclusion criteria were no diagnosis of MS and not being over the age of 18 .

\section{Data Collection and Measurements}

An online survey on the broad topic of 'psychological support in MS' was conducted during the COVID-19 lockdown period, from midMarch to mid-August, 2020. Data were collected on the experiences of psychological support as well as psychological variables listed below relevant to the present study. Participants were recruited via various MS organisations globally as well as MS social media groups via e-newsletters and/or social media posts with a link to the online survey.

Participants were asked to provide demographic information such as age, gender, location, employment status, type of MS and time since diagnosis. Additionally, participants were requested to rate on a Likert-type scale from 1 to 5 how bothered they were by broad clusters of common MS symptoms including motor, such as mobility issues; sensory, such as numbness and tingling; cognitive, such as problems with memory and attention; and psychological symptoms, such as anxiety and depression; as well as fatigue. As variables that could potentially interact with the impact of COVID-19, they were also investigated in this present study. These data are presented in Table 1.

Participants were also asked to indicate their overall self-perceived level of impact of the current COVID-19 pandemic on them generally, including their MS (positive, such as spending more time with family; neutral, that is little or no impact; or negative, such as anxieties about MS medications). They were then asked to briefly provide some insight into why they reported this level of impact.

As part of the broader survey, participants were also asked to indicate their level of concern regarding factors that are often reported to be 
Table 1 Participant characteristics

\begin{tabular}{|c|c|}
\hline & Frequency \\
\hline \multicolumn{2}{|l|}{ Age } \\
\hline $20-39$ & 83 \\
\hline $40-59$ & 183 \\
\hline $60+$ & 58 \\
\hline \multicolumn{2}{|l|}{ Gender } \\
\hline Male & 51 \\
\hline Female & 273 \\
\hline \multicolumn{2}{|l|}{ Location } \\
\hline UK & 131 \\
\hline USA & 111 \\
\hline Other & 82 \\
\hline \multicolumn{2}{|c|}{ Living arrangements } \\
\hline Alone & 55 \\
\hline With others & 269 \\
\hline \multicolumn{2}{|c|}{ Employment status } \\
\hline Employed & 141 \\
\hline Unemployed & 183 \\
\hline \multicolumn{2}{|l|}{ MS type } \\
\hline RRMS & 246 \\
\hline Progressive & 59 \\
\hline CIS & 19 \\
\hline \multicolumn{2}{|c|}{ Years diagnosed with MS } \\
\hline $0-5$ & 134 \\
\hline $6-10$ & 60 \\
\hline $11+$ & 130 \\
\hline \multicolumn{2}{|c|}{ Fatigue symptom? } \\
\hline Yes & 301 \\
\hline No & 23 \\
\hline \multicolumn{2}{|c|}{ Sensory symptoms? } \\
\hline Yes & 313 \\
\hline No & 11 \\
\hline \multicolumn{2}{|c|}{ Motor symptoms? } \\
\hline Yes & 278 \\
\hline
\end{tabular}

Table 1 continued

\begin{tabular}{lc}
\hline & Frequency \\
\hline No & 46 \\
Cognitive symptoms? & \\
Yes & 278 \\
No & 46 \\
Psychological symptoms? & \\
Yes & 291 \\
No & 33 \\
\hline
\end{tabular}

$R R M S$ relapsing-remitting multiple sclerosis, CIS clinically isolated syndrome

impacted by a diagnosis of MS, such as anxiety, loss, impact on relationships and life goals, on a Likert scale of 1-3.

\section{Data Analysis}

Quantitative data were analysed using IBM Statistical Package for the Social Sciences (SPSS) software version 25.0. A multinomial regression was conducted to identify which variables, if any, could predict a negative impact of the COVID-19 pandemic. A series of one-way ANOVAs were performed to explore differences in levels of MS-related concern and symptom bother between the three different levels of COVID-19 impact. Due to the uneven distribution between the three COVID-19 impact groups, Welch's F was used as a more robust alternative. Qualitative data briefly describing reasons for the reported level of COVID-19 impact were analysed by identifying general themes and presented as frequencies and percentages. These were then compared between the three different levels of overall COVID-19 impact (positive, neutral or negative).

\section{Ethical Considerations}

Ethical approval was obtained from the University of Reading Ethics Committee (approval number: UREC 16/30). This study was performed in accordance with the Helsinki 
Declaration of 1964 and its later amendments. Participants provided written informed consent digitally before completing the survey.

\section{RESULTS}

\section{COVID-19 Impact}

We found that $36 \%(n=117)$ endorsed a negative COVID-19 impact; however, $16 \%(n=52)$ reported a positive impact, and the most common response was a neutral impact, $48 \%$ $(n=155)$. This suggests that for just under half of the people in this sample, the overall impact of COVID-19 has been largely neutral. Just over a third have experienced negative consequences of COVID-19, with the least number of people reporting a positive impact. Despite the sample residing in different countries, the pattern of results was similar in the countries from where the largest number of participants are represented ( $n>100$; UK and USA), with most reporting a neutral impact (42\%, UK; 55\%, USA), closely followed by a negative impact (41\%, UK; $32 \%$, USA) and the least common impact was positive (17\%, UK; 13\%, USA).

\section{Predicting COVID-19 Impact}

To predict those negatively impacted by the COVID-19 lockdown, demographic and MS-related variables were entered into a multinomial regression.

The full model, including age, gender, location, employment status, living arrangements, whether taking medication for a mood disorder or not, type of MS, years diagnosed with MS and presence (or absence) of symptoms provides a statistically significant improvement in fit over a null model $(\chi 2(30)=74.04, p<0.001)$.

Age, type of MS and self-reported presence of psychological symptoms (such as anxiety and depression) were the significant predictors in the model where lower age predicted increased likelihood of reporting a negative response than a neutral $(\beta=1.07, \mathrm{SE}=0.01, p<0.001)$ or positive $(\beta=1.04, \mathrm{SE}=0.02, p<0.05)$ response. People with progressive types of MS were more likely to report a negative response than a positive one $(\beta=0.10), \mathrm{SE}=0.94, p<0.05)$, and those who reported having psychological symptoms in relation to their MS were also more likely to report a negative impact than a neutral $(\beta=8.02), \mathrm{SE}=.80, p<0.01)$ or positive impact $(\beta=9.11, \mathrm{SE}=0.90, \quad p<0.05)$. Gender, location, employment status, living arrangements, and taking mood disorder medication were not significant predictors of level of COVID-19 impact in the model.

\section{Relationship Between MS Concerns and Symptom Bother and COVID-19 Experience}

When investigating levels of MS-related concerns between the three different levels of COVID-19 impact, statistically significant main effects were observed between group means as determined by a one-way ANOVA for all types of MS-related concern examined (anxiety, Welch's $F(2,143.58=14.26, p<0.01$; sadness, Welch's $F(2,141.05)=10.73, p<0.01 ;$ loss, Welch's $F(2,141.88)=10.49, p<0.01$; coping, Welch's $F(2,136.52)=12.55, p<0.01$; uncertainty, Welch's $F(2,140.61)=6.05, p<0.01$; control, Welch's $F(2,141.26)=4.75, p<0.05$; function, Welch's $F(2,139.44)=8.54, p<0.01$; disease progression, Welch's $F(2$, $137.82)=8.36, p<0.01$; fatigue, Welch's $F(2$, $139.12)=7.93, \quad p<0.01 ; \quad$ lifestyle change, Welch's $F(2,135.92)=8.39, p<0.01$; life goals, Welch's $F(2,136.67)=7.93, p<0.01$; family impact, Welch's $F(2,140.18)=4.42, p<0.05$; relationship impact, Welch's $F(2$, 136.53) $=7.68, p<0.01$.

Table 2 shows the means for each concern variable at each level of COVID-19 impact. In all cases, the mean level of concern for each variable was significantly higher amongst those who reported a negative impact compared to those who reported a neutral and/or positive impact. Three patterns of response were evident. First, the negative group had significantly higher concerns (about anxiety, sadness, loss, coping, fatigue, lifestyle change, life goal impact and relationship impact) than both the neutral and positive groups, which were similar. 
Table 2 Means of MS related concern ratings (1-3) and symptom bother (1-5) for each level of COVID-19 impact (where a higher number indicates a higher rated level of concern)

\begin{tabular}{|c|c|c|c|c|c|}
\hline & Negative & & Neutral & & Positive \\
\hline \multicolumn{6}{|l|}{ Concern ratings } \\
\hline Feelings of anxiety & 2.38 & $>$ & 1.92 & $=$ & 1.98 \\
\hline Feeling low/sad & 2.33 & $>$ & 1.93 & $=$ & 2.00 \\
\hline Feelings of loss & 2.31 & $>$ & 1.91 & $=$ & 2.00 \\
\hline Lack of resources to cope & 2.21 & $>$ & 1.75 & $=$ & 1.77 \\
\hline Uncertainty about the future & 2.51 & $>$ & 2.23 & $<$ & 2.35 \\
\hline Lack of control of my MS & 2.42 & $>$ & 2.15 & $<$ & 2.31 \\
\hline Ability to function as I want & 2.62 & $=$ & 2.39 & $>$ & 2.21 \\
\hline Worry about MS disease progression & 2.57 & $>$ & 2.28 & $<$ & 2.44 \\
\hline Fatigue & 2.69 & $>$ & 2.38 & $=$ & 2.40 \\
\hline Having to make lifestyle changes & 2.38 & $>$ & 2.08 & $=$ & 2.00 \\
\hline Impact on my life goals & 2.52 & $>$ & 2.21 & $=$ & 2.21 \\
\hline Worry about impact on my family & 2.48 & $>$ & 2.23 & $<$ & 2.35 \\
\hline Impact on relationships with others & 2.45 & $>$ & 2.17 & $=$ & 2.10 \\
\hline \multicolumn{6}{|l|}{ Symptom bother } \\
\hline Motor symptoms & 2.90 & $=$ & 2.67 & $=$ & 2.29 \\
\hline Sensory symptoms & 3.51 & $=$ & 3.17 & $=$ & 3.13 \\
\hline Cognitive symptoms & $2.94^{\times}$ & $=$ & 2.57 & $=$ & 2.23 \\
\hline Psychological symptoms & 3.44 & $>$ & 2.67 & $=$ & 2.56 \\
\hline Fatigue & 3.91 & $>$ & 3.35 & $=$ & 3.08 \\
\hline
\end{tabular}

Symbols between ratings depict direction of statistical significance $(p<0.05)$.

${ }^{\mathrm{X}}$ Significant difference between negative and positive $(p<0.05)$

Second, both the negative and neutral groups had significantly higher concern (about ability to function) than the positive group. Third, the negative and positive groups both showed statistically higher levels of concern (about uncertainty, control, disease progression and family impact) than the neutral group.

To examine differences in level of symptom bother experienced between the different levels of COVID-19 impact (see Table 2), a further series of one-way ANOVAs were conducted. Results indicate no significant differences between the level of bother experienced by sensory and motor symptoms between each level of COVID-19 impact. However, statistically significant differences were observed for levels of current bother for fatigue (Welch's $F(2,131.40)=7.27, \quad p<0.01), \quad$ psychological symptoms $\quad($ Welch's $\quad F(2,136.60)=11.69$, $p<0.01$ ) and cognitive symptoms (Welch's $F(2,146.75)=4.31, p<0.05)$.

As illustrated in Table 2, the means for each symptom bother variable between each level of COVID-19 impact was higher in the negative impact group. For psychological symptoms and fatigue, this was significantly higher than both neutral and positive impact groups, and for 
cognitive symptoms the mean was significantly higher than the positive group alone.

\section{The COVID-19 Pandemic Experience for MS Patients}

Figure 1 illustrates a breakdown of the broad themes identified as given in the responses provided by participants in the open-ended question, as well as the types of responses that were included in each theme in brackets.

All participants who reported a negative impact $(n=117)$ stated some form of health- related concern for themselves or others. In particular, concerns like facing uncertainty, reduced immune function and worsening symptoms $(n=76,65 \%)$ and interruptions to treatment in terms of medication and/or contact with medical professionals $(n=44,38 \%)$ were the top contributors to a negative COVID19 impact.

Regarding those who reported a neutral impact, a large percentage of people stated no change ( $n=89,57 \%)$ either due to still being able to work, or because they already felt isolated. Like those who reported a negative

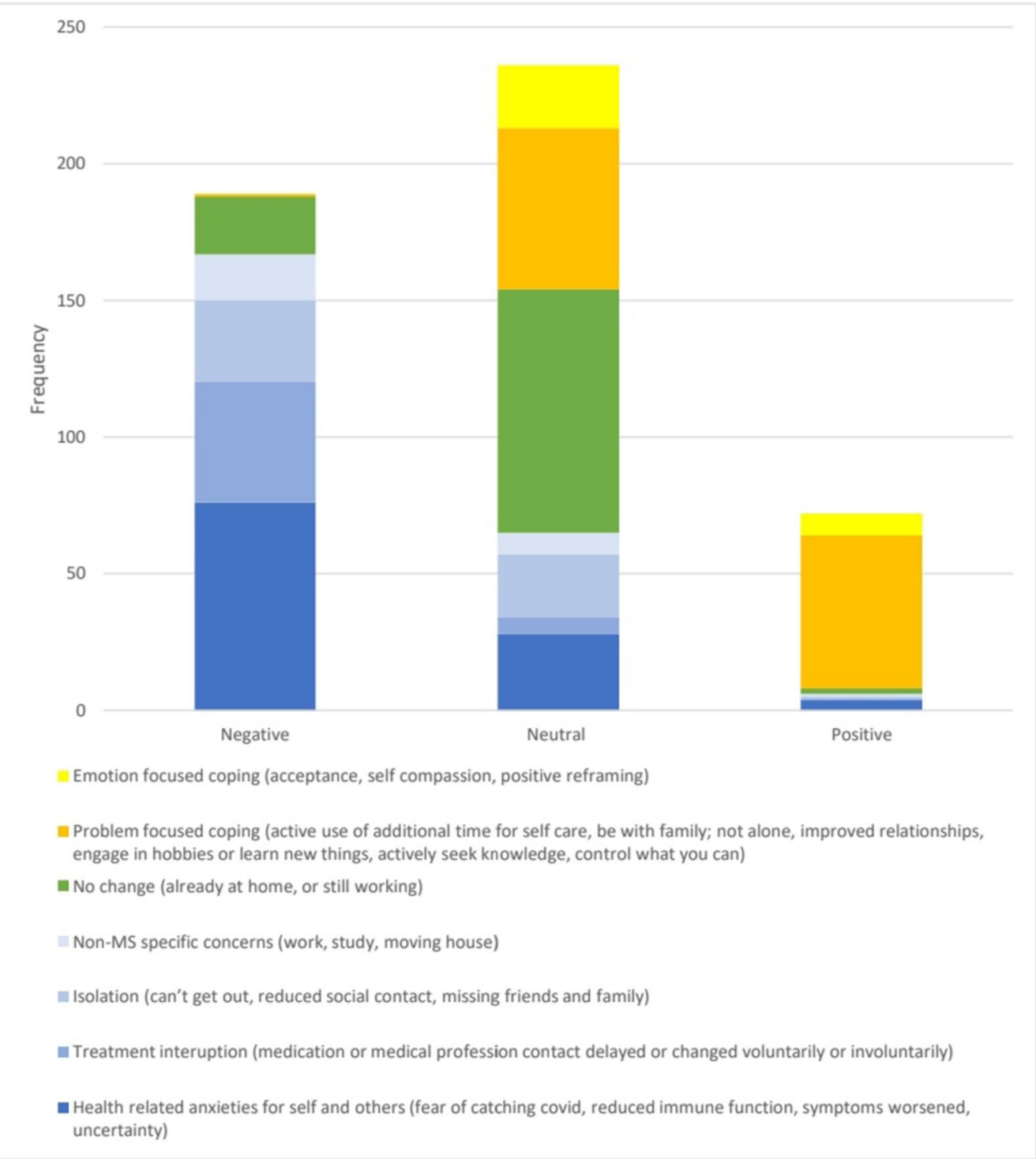

Fig. 1 Frequency of reasons stated for overall level of COVID-19 impact 
impact, heightened levels of concern in relation to fear for self and others, treatment interruption, isolation and non-MS related worries featured in $42 \%(n=65)$ of this group. However, unlike the negative impact group, there was also evidence to suggest that certain coping strategies were instrumental in neutralising the impact of some of the negative consequences of the COVID-19 pandemic. Both problem-focused $(n=59,38 \%)$ and emotion-focused approaches $(n=23,15 \%)$ were utilised by this group to this effect. For example, acceptance: 'I am anxious about Covid 19 but that is settling... I am doing the sensible thing and am now accepting 2020 is the year that never was' and positive reframing: 'My anxiety levels are high from risk of becoming ill...Working from home has had a hugely positive impact on my well-being and my stamina to walk has increased to a great level-am healthier than I've been in a long time and stress is definitely reduced' are amongst the emotion-focused strategies used by this group in response to any negative impact experienced.

Like the neutral group, $15 \%$ of the positive group also reported using emotion-focused strategies. However, the dominating coping approach used by this group was the use of problem-focused strategies $(88 \%, n=46)$. Active problem-focused strategies were used the most frequently by making use of the additional time gained as a result of the lockdown to be with family ( $n=27,52 \%)$ : 'I'm not the easiest person to be around because of my MS so in isolation with my family allows me time to mend bridges', actively choosing to engage in self-care by slowing the pace down and resting $(n=14$, 27\%): 'I am currently working from home which has allowed me more time and flexibility to look after myself, including my diet, exercise regimes and stress management', or engaging in existing or new hobbies $(n=11,21 \%)$ : 'I started doing yoga and prepared more meals at home'.

\section{DISCUSSION}

This study adds further understanding to the experience of the COVID-19 pandemic in MS patients by highlighting which factors contribute to this experience. By further exploring the net effect of COVID-19 consequences on individuals and factors most prevalent in each impact group (negative, neutral or positive) we provide a more detailed insight into the experience amongst MS patients.

Overall, our hypothesis was supported with the most common experience being neutral, as reported by just under half of our sample. This was closely followed by over a third of participants who reported a negative impact; they had greater levels of MS-related concerns and MS symptom bother from psychological, cognitive and fatigue symptoms. Demographic variables that predicted this negative impact were younger age, progressive types of MS and the presence of psychological symptoms. This is consistent with previous research $[9,10]$ that found those of a younger age to be more vulnerable to the negative effects of COVID-19 in the form of increased anxiety at this time. However, the present study also found that location, gender, employment status, time since diagnosis and mood disorder medication use were not significant predictors of negative impact in the model which provides some evidence to the contrary that gender, in this case, has no relationship with the self-reported impact of COVID-19 [5, 8].

It was also found that MS-related concerns (such as feelings of loss, impact on relationships and disease progression) and bother related to psychological and cognitive symptoms and fatigue were higher amongst those who reported a negative impact. In this case, it may be that since these were MS-specific outcomes, it naturally matched the concerns that might be had amidst the COVID-19 pandemic and therefore might be expected that higher levels of these factors related to a negative pandemic experience. In the case of symptom bother, this would also lend support to previous studies that have identified MS care being impacted at this time [15].

On the other hand, it may seem surprising to find that levels of bother related to motor symptoms were statistically similar between the three impact groups despite it being found that people with progressive types of MS, typically 
characterised by motor difficulties, were more likely to report a negative impact. However, this could possibly be explained by examining coping approaches as expressed in the open-ended responses. For example, an interesting finding reflecting this is noted when examining concern about disease progression. In this case, scores were statistically similar between those who rated a negative and positive COVID-19 impact, but higher than those who rated a neutral impact, indicating a higher level of concern for disease progression amongst both those who reported negative and positive COVID-19 impacts compared to those who reported a neutral impact. When taking into account the open-ended responses in the neutral category, it would appear that coping strategies exhibited by those in the neutral category could be responsible for the neutralising effect. In this way, a potentially negative impact could be reduced to something more manageable and therefore yielding a neutral effect. This particular example provides insight into how coping strategies may be responsible for different experiences explained by demographic factors, MS related concerns and symptom bother between the impact groups.

Furthermore, heightened levels of health-related concern were qualitatively identified as the most common experience regardless of what level of COVID-19 impact was reported. This provides further support to previous claims suggesting heightened levels of anxiety are being experienced in people with MS during the COVID-19 pandemic [5]. Further qualitative outcomes in the present study also provide insight into factors that may contribute to understanding why other studies have found evidence to the contrary, that anxiety levels have not increased at this time [5, 7]. People who reported a neutral impact, despite commonly reporting health-related concerns, also reported the use of both problem- and emotionfocused coping strategies that seemingly neutralised the negative effects of COVID-19. This points towards coping as a mechanism that may be a factor that contributes to previous discrepancies in findings.

Additionally, the present study presents evidence that active, problem-focused coping strategies are utilised more frequently by those who report a positive impact and by some who report a neutral impact. This supports previous claims that active coping strategies could be related to more positive outcomes [11], and in this case, a positive or neutral COVID-19 impact. These coping methods are largely absent amongst those who reported a negative impact. This finding in particular may provide insight into any discrepancies that have been found in recent research between whether anxieties are increasing for people with MS or not at this time $[5,6]$. This may also be an indication that, for some people with MS who are well accustomed to dealing with uncertainties and limitations that arise as a result of their illness, they are already equipped with skills to cope effectively with yet another situation that stimulates feelings of uncertainty.

Dividing COVID-19 impact into three distinct categories-negative, neutral and positive-has allowed for a more detailed examination into factors that play a significant role in the negative experience of the COVID-19 pandemic, whilst taking into account self-reported overall impact and using this as a basis to compare experiences at this time. The direct approach taken allowed us to understand the overall perceived COVID-19 impact in people with MS as well as the relevant contributing factors. However, the costs of taking this approach arguably led to some potential challenges. For example, the use of self-report meant that information such as type of MS could not be clinically verified. The opportunistic nature of type of sample itself may be responsible for some level of bias in terms of how and from where participants were recruited in this global sample. Three-point Likert scales were considered appropriate for the nature of this study, but may arguably result in limited granularity. Similarly, a single question to assess overall impact was the most suitable way of allowing participants to self-assess all factors relevant to them individually and reflect this in a single overall response. Participants were free to provide information on any factors that were meaningful in making their choice of overall impact; however, more systematic scrutiny of a range of specific factors such as exposure to 


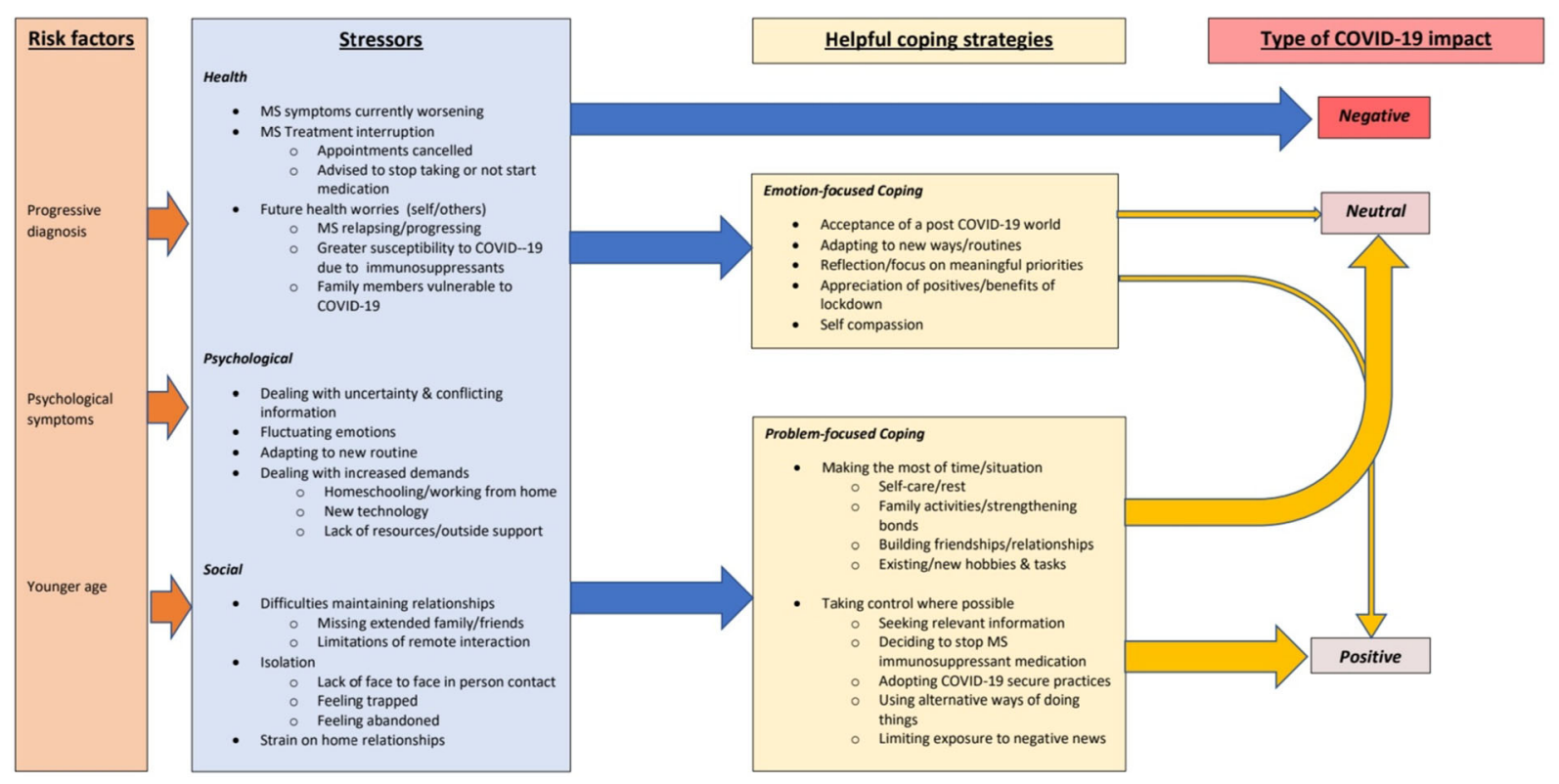

Fig. 2 Summary model of COVID-19 impact in MS patients

COVID-19 and level of disability was outside the scope of the current study.

This study was strengthened by the strong sample size and has shown that the detrimental impact of COVID-19 can be predicted by a progressive diagnosis of MS, being younger and having psychological symptoms. Furthermore, our novel and direct approach allowed participants to explore the factors that contributed to their experience based on what is most meaningful to them. This insightful qualitative data allowed us to generate a model of pandemic impact in MS patients (see Fig. 2) that may also be applicable to other chronic neurological conditions with similar characteristics.

Our summary model in Fig. 2 identifies key risk factors, stressors and coping strategies that determine the perceived impact of a pandemic and its repercussions on everyday life. In particular, it highlights the types of coping strategies utilised that could be applied in a practical way to encourage support those in the more vulnerable sub-group to promote a more positive or at least neutral impact. Given the nature of chronic illness more generally, such approaches are also likely to be useful for targeting support in similar neurological conditions.

\section{CONCLUSIONS}

These findings suggest that younger patients, those with a progressive diagnosis and those with psychological symptoms, such as anxiety and depression, are particularly vulnerable to the negative effects of a COVID-19-induced lockdown, and may therefore benefit from further support. With the rapidly evolving nature of healthcare delivery, alternatives to face-toface modes of support may be helpful to ameliorate the negative pandemic-induced repercussions on daily life and well-being. Coping strategies appear to be related to the overall COVID-19 impact experienced, with active, problem-focused approaches featuring most frequently amongst the positive and neutral impact groups, with evidence of some emotionfocused approaches being used by these groups as well. The practical strategies summarised in Fig. 2 have been helpful in our sample. Taken together, our data serve as a pragmatic well-being guide, as well as a timely call to action for more MS support in a post COVID-19 world, particularly in those whom we have identified as being most vulnerable. 


\section{ACKNOWLEDGEMENTS}

We wish to thank all the MS organisations who support our work-MSTrust, MS-UK, OMS, MSRA, MS Ireland, MS New Zealand and MS Canada, amongst others-as well as the various social media pages and their members who participated in this study.

Funding. The Rapid Service Fee was funded by The University of Reading. This research received no specific grant from any funding agency in the public, commercial, or not-forprofit sectors.

Authorship. All named authors meet the International Committee of Medical Journal Editors (ICMJE) criteria for authorship for this article, take responsibility for the integrity of the work as a whole, and have given their approval for this version to be published.

Authors' Contributions. Conception and design: AH, HM; Data acquisition: HM, AH; Statistical analysis and interpretation: $\mathrm{HM}, \mathrm{AH}$; Drafting manuscript and critical revision: HM, $\mathrm{AH}$.

Disclosures. Hannah Morris-Bankole and Aileen Ho declare that they have no conflict of interest. Aileen Ho is a member of the journal's Editorial Board.

Compliance with Ethics Guidelines. Ethical approval was obtained from the University of Reading Ethics Committee (approval number: UREC 16/30). Participants provided written informed consent digitally before completing the survey. This study was performed in accordance with the Helsinki Declaration of 1964, and its later amendments.

Data Availability. The data sets generated and/or analysed during the current study are available from the corresponding author.

Open Access. This article is licensed under a Creative Commons Attribution-NonCommercial 4.0 International License, which permits any non-commercial use, sharing, adaptation, distribution and reproduction in any medium or format, as long as you give appropriate credit to the original author(s) and the source, provide a link to the Creative Commons licence, and indicate if changes were made. The images or other third party material in this article are included in the article's Creative Commons licence, unless indicated otherwise in a credit line to the material. If material is not included in the article's Creative Commons licence and your intended use is not permitted by statutory regulation or exceeds the permitted use, you will need to obtain permission directly from the copyright holder. To view a copy of this licence, visit http://creativecommons.org/licenses/by$\mathrm{nc} / 4.0 /$.

\section{REFERENCES}

1. Pfeferrbaum B, North CS. Mental health and the Covid-19 pandemic. N Engl J Med. 2020. https:// doi.org/10.1056/NEJMp2008017.

2. Boeschoten RE, Braamse AMJ, Beekman ATF, et al. Prevalence of depression and anxiety in multiple sclerosis: a systematic review and meta-analysis. J Neurol Sci. 2017;372:331-41. https://doi.org/10. 1016/j.jns.2016.11.067.

3. Moghadasi AN. One aspect of coronavirus disease (COVID-19) outbreak in Iran: high anxiety among MS patients. Mult Scler Relat Disord. 2020;41: 102138.

4. Rezaeimanesh N, Sahraian MA, Naser MA. Evaluation of the opinion of patients with multiple sclerosis on the outcomes of catching COVID-19 and its effects on the MS symptoms. Basic Clin Neurosci. 2020;11:201-6. https://doi.org/10.32598/bcn.11. covid19.2520.1.

5. Radulovic L, Erakovic J, Roganovic M. Attitudes of patients with relapsing-remitting form of multiple sclerosis using disease-modifying drugs in Montenegro regarding COVID-19 pandemic. Mult Scler Relat Disord. 2020. https://doi.org/10.1016/j. msard.2020.102380.

6. Stojanov A, Malobabic M, Milosevic V, et al. Psychological status of patients with relapsing-remitting multiple sclerosis during coronavirus disease2019 outbreak. Mult Scler Relat Disord. 2020. https://doi.org/10.1016/j.msard.2020.102407. 
7. Capuano R, Altieri M, Bisecco A, et al. Psychological consequences of COVID-19 pandemic in Italian MS patients: signs of resilience? J Neuro. 2020. https:// doi.org/10.1007/s00415-020-10099-9.

8. Vogel AC, Schmidt H, Loud S, McBurney R, Mateen FJ. Impact of the COVID-19 pandemic on the health care of $>1000$ People living with multiple sclerosis: a cross-sectional study. Mult Scler Relat Disord. 2020;46:102513. https://doi.org/10.1016/j. msard.2020.102512.

9. Talat F, Ramadan I, Aly S, Hamdy E. Are multiple sclerosis patients and their caregivers more anxious and more committed to following the basic preventive measures during the COVID-19 pandemic? Mult Scler Relat Disord. 2020;46:102580. https:// doi.org/10.1016/j.msard.2020.102580.

10. Alschuler KN, Roberts MK, Herring TE, Ehde DM. Distress and risk perception in people living with multiple sclerosis during the early phase of the COVID-19 pandemic. Mult Scler Relat Disord. 2020;47:102618. https://doi.org/10.1016/j.msard. 2020.102618 .

11. Pakenham KI. Adjustment to multiple sclerosis: application of a stress and coping model. Health Psychol. 1999;18:383-92.

12. Sormani MP, Italian Study Group on COVID-19 infection in multiple sclerosis. An Italian programme for COVID-19 infection in multiple sclerosis. Lancet Neuro. 2020;19:481-2. https://doi.org/ 10.1016/S1474-4422(20)30147-2.
13. Giovannoni G. Anti-CD20 immunosuppressive disease-modifying therapies and COVID-19. Mult Scler Relat Disord. 2020;41:102135. https://doi.org/ 10.1016/j.msard.2020.102135.

14. Seery N, Li V, Nguyen AL, Roos I, et al. Evaluating the perspective of patients with MS and related conditions on their DMT in relation to the COVID19 pandemic in one MS centre in Australia. Mult Scler Relat Disord. 2020;46:102516. https://doi.org/ 10.1016/j.msard.2020.102516.

15. Sastre-Garriga J, Tintoré M, Montalban X. Keeping standards of multiple sclerosis care through the COVID-19 pandemic. Mult Scler J. 2020. https:// doi.org/10.1177/1352458520931785.

16. Haji Akhoundi F, Sahraian MA, Naser Moghadasi AN. Neuropsychiatric and cognitive effects of the COVID-19 outbreak on multiple sclerosis patients. Mult Scler Relat Disord. 2020;2020(41):102164.

17. Wilski M, Tomczak M. Comparison of personal resources in patients who differently estimate the impact of multiple sclerosis. Ann Behav Med. 2017;51(2):179-88. https://doi.org/10.1007/ s12160-016-9841-5.

18. Samios C, Pakenham KI, O'Brien J. A dyadic and longitudinal investigation of adjustment in couples coping with multiple sclerosis. Ann Behav Med. 2015;49(1):74-83. https://doi.org/10.1007/s12160014-9633-8. 